\title{
Location specificity in response selection processes for visual stimuli
}

\author{
HAGIT MAGEN and ASHER COHEN \\ The Hebrew University, Jerusalem, Israel
}

\begin{abstract}
We used the psychological refractory period paradigm, in which participants respond to two successive tasks (T1 and T2). We created in T2 spatial and color Simon effects, known to be caused by response selection processes. Previous studies in which the spatial Simon effect was manipulated in T2 showed that this effect was underadditive, with stimulus onset asynchrony (SOA) between the targets for T1 and T2. In Experiment1, we replicated these results with two versions of the spatial Simon effect. In contrast, in Experiment 2 we manipulated two versions of a color Simon effect, revealing an additive relation between the color Simon effect and SOA. These results suggest that the underadditivity obtained with the spatial Simon effect is due to its spatial nature, and that space may play a unique role in response selection processes.
\end{abstract}

A long-standing debate in vision concerns the role of spatial location in visual attention. Several researchers have suggested that spatial location has a special role in attentional selection (e.g., Posner, Snyder, \& Davidson, 1980; Tsal \& Lavie, 1993). Others have proposed that selection by spatial location is similar to selection by other features, such as color and shape (e.g., Baylis \& Driver, 1992; see Lamy \& Tsal, 2001, for a review). This debate has been focused exclusively on attentional selection. We provide evidence that location may play a unique role in response selection processes for visual stimuli. We use the well-known Simon interference task for this purpose.

According to its original definition, the Simon effect involved only spatial interference (Craft \& Simon, 1970). For example, participants are asked to respond to a nonspatial stimulus property (e.g., color) with spatially arranged response buttons (e.g., red and green targets are assigned to a left button and a right button, respectively). The Simon effect refers to the findings that in a compatible condition, in which the target appears on the same side as its assigned response button (e.g., the red target appears on the left side), reaction time (RT) is faster than it is in an incompatible condition, in which the target appears on the opposite side of the corresponding response button (e.g., the red target appears on the right side). Note that the target location is irrelevant to the task, and yet it interferes with performance. As Kornblum, Hasbroucq, and Osman (1990) pointed out, the Simon effect can be generalized to nonspatial features as well, involv-

This study was funded by a grant from the Israel Science Foundation to A.C. We thank Tram Neill, Bernhard Hommel, and Yehoshua Tsal for their helpful comments on an earlier version of this article. Correspondence concerning this article should be sent to A. Cohen or H. Magen, Department of Psychology, The Hebrew University, Jerusalem 91905, Israel (email:msasher@mscc.huji.ac.il ormsmagen@mscc.huji.ac.il). ing interference between an irrelevant feature of the target and a feature of the required response.

It is well established that the Simon effect is caused by response selection processes (Lu \& Proctor, 1995). Consequently, resolution of the Simon conflict must also involve response selection processes. We use the psychological refractory period (PRP) paradigm to demonstrate that the resolution of the Simon conflict is fundamentally different for spatial and for nonspatial interference.

In the PRP paradigm, participants make speeded responses to two tasks whose input is presented in succession. Participants must respond to the first task (T1) before they respond to the second task (T2). The stimulus onset asynchrony (SOA) between the presentation of the targets in T1 and T2 is manipulated. Typically, RTs for T1 are minimally affected by SOA, but those for T2 get progressively longer with smaller SOAs (see, e.g., Welford, 1952). This pattern of results is known as the PRP effect.

The PRP paradigm is often investigated with the locus-of-slack method (see, e.g., Schweickert, 1980), which involves a manipulation of the difficulty of specific processing stages (e.g., perception, response selection, response execution) for T2. The locus-of-slack method is used to examine whether the PRP effect is caused by an inherent limitation (i.e., a bottleneck) in the simultaneous use of one of these stages for two tasks. If two tasks require this bottleneck stage simultaneously, as might be the case in the small-SOA conditions of the PRP task, one of them (T2 in the PRP paradigm) is postponed until the other is finished with it. This method yields clear predictions (Figure 1). If the manipulated stage precedes the bottleneck stage, an underadditive pattern will emerge, in which the difference between the easy and difficult conditions will be apparent in the large SOAs but will be reduced and perhaps even eliminated in the small SOAs. If, however, the manipulation involves a 


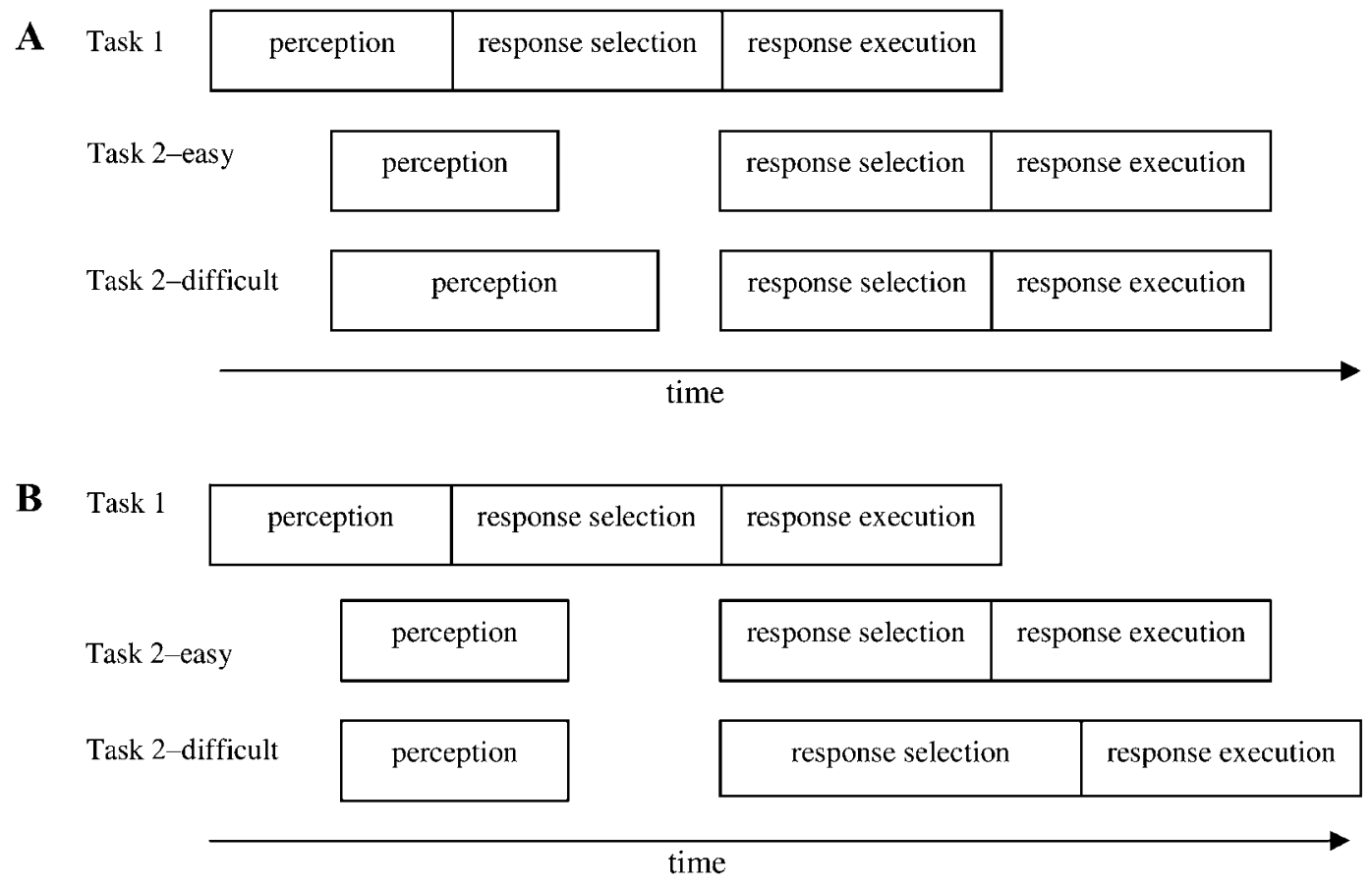
Figure 1. (A) An illustration of underadditivity in the psychological refractory period (PRP) paradigm. (B) An
illustration of additivity in the PRP paradigm.

stage at or following the bottleneck stage, the difference between the easy and difficult conditions will be unaffected by SOA (i.e., the relation between the SOA and $\mathrm{T} 2$ manipulation is additive).

In many studies, manipulating the difficulty of the perception stage of $\mathrm{T} 2$ produced underadditivity with the SOA (e.g., Pashler \& Johnston, 1989). In contrast, manipulating the response selection difficulty of T2 produced additivity with the SOA (see, e.g., McCann \& Johnston, 1992). The logic of the locus-of-slack method implies that the response selection stage is a fixed bottleneck for dual task performance, leading to the formation of the response selection bottleneck model (see Pashler, 1994, for a review; see Cohen \& Magen, 2004, and Meyer \& Kieras, 1997, for alternative interpretations).

A notable exception to these findings involves the spatial Simon effect. McCann and Johnston (1992) used the spatial Simon effect as a T2 manipulation in the PRP paradigm. Although the Simon effect involves response selection processes (see, e.g., Lu \& Proctor, 1995), McCann and Johnston found underadditivity of the effect in the PRP paradigm. Explanations for this finding (Lien \& Proctor, 2000; McCann \& Johnston, 1992) will be described later.

We examined whether the underadditivity of the Simon effect is unique to its spatial version or will also be observed with a color Simon task. We are not aware of a previous report of a color Simon effect. We chose to compare color with space because both are basic visual features and because color and space were often compared in the attention literature mentioned earlier (see, e.g., Tsal \& Lavie, 1993). To create comparable color and spatial Simon effects, we had our participants respond by touching one of two peripheral squares on a touch screen. In our color Simon task, the participants responded to the identity of a central colored shape by touching one of two colored squares on a touch screen. The irrelevant color of the shape could match the color of the square that was assigned to that shape (the compatible condition) or the color of the alternative response (the incompatible condition), creating the color Simon effect. ${ }^{1}$ In our spatial Simon task, the participants responded to the identity of a peripheral shape, located either on the right or on the left, by touching one of two peripheral squares. The shape location, though irrelevant to the task, matched or mismatched the location of the square. In pilot studies, we obtained robust Simon effects for both versions of the Simon task. In our main experiments, we embedded these two versions in the form of a T2 in the PRP paradigm.

\section{EXPERIMENT 1}

The goal of Experiment 1 was to replicate the underadditivity of the spatial Simon PRP experiment using a touch screen to gather responses. The targets were the letters $\mathrm{X}$ and $\mathrm{O}$, presented on the left or on the right side of the screen. Participants responded by touching one of two squares that appeared on the bottom left and bottom 
right sides of the screen. We expected to find an underadditive interaction between the spatial Simon and PRP effects (see, e.g., McCann \& Johnston, 1992).

\section{Method}

Participants. Twenty-five students from The Hebrew University participated (for course credit or payment) in two separate sessions. The participants reported having normal or corrected-to-normal vision.

Apparatus and Stimuli. The participants sat in a dimly lit room and viewed the display from an approximate distance of $40 \mathrm{~cm}$. The stimuli were presented on a Philips color touch screen controlled by a Pentium microcomputer.

The stimulus for $\mathrm{T} 1$ was a computer-generated tone presented at 300 or $900 \mathrm{~Hz}$, lasting $150 \mathrm{msec}$. The T2 target - either X or O - appeared on the left or the right side of the screen. The letters subtended approximately $1.43^{\circ}$ of visual angle in length and width, with luminance of approximately $20 \mathrm{~cd} / \mathrm{m}^{2}$ against a dark background $\left(1 \mathrm{~cd} / \mathrm{m}^{2}\right)$. The center-to-center distance between the letters and a central fixation point was $7.55^{\circ}$. The participants responded to the tone by pressing one of two buttons mounted on a response board interfaced with the computer. The response to the shapes required that the participant touch one of two squares (both gray in Version 1, one red and one green in Version 2) on the screen, positioned approximately $7.97^{\circ}$ of visual angle (center to center) below the locations of the target letters. The squares' sides subtended approximately $4.15^{\circ}$ of visual angle.

Design. Each trial consisted of a tone task, in which the participants discriminated between high and low tones, and a shape task, which required a discrimination between $\mathrm{X}$ and $\mathrm{O}$. The participants responded to $\mathrm{X}$ and to $\mathrm{O}$ by touching the left and right squares, respectively. Each letter appeared equally often on the right and left sides, creating two conditions. In the compatible condition, the target appeared on the same side as its assigned response, whereas in the incompatible condition the target appeared on the opposite side of its assigned response.

The tone and the letter were separated by four different SOAs $(50,150,300$, and $900 \mathrm{msec})$, each used on $25 \%$ of the trials. Each of the tones and letters appeared in $50 \%$ of the trials. This 4 $(\mathrm{SOA}) \times 2$ (auditory target) $\times 2$ (second task target) $\times 2$ (compatibility) factorial design resulted in 32 different displays.

Each session included eight experimental blocks of 96 trials. The participants received three types of practice in the first session, prior to the experimental blocks. First, they performed 30 practice trials for the tone task only, then a demonstration of 16 trials of both tasks, and finally, a practice block of 96 trials.

Procedure. Each trial began with the presentation of an achromatic asterisk, which served as a fixation point. After $250 \mathrm{msec}$, it was replaced by a 150 -msec tone. Following a variable SOA, a letter appeared on either the left or the right side of the screen. The participants were instructed to respond first to the tone and then to the letter. The letter remained on the screen until responses were registered for both tasks. Following incorrect responses, an error message was presented on the screen for $500 \mathrm{msec}$, noting whether the error was on T1, T2, or both. In either case, the intertrial interval was $1,500 \mathrm{msec}$. The participants were asked to respond as rapidly and as accurately as possible on both tasks. The need for a speeded response to the tone was further emphasized to discourage the participants from grouping the two responses into one unit (Pashler \& Johnston, 1989).

\section{Results and Discussion}

Data from the first session were considered practice and will not be reported. For each participant, we first removed all RTs greater than $3,000 \mathrm{msec}$. Of the remaining trials, all RTs exceeding three standard deviations from the mean for each condition were removed. Correct responses for $\mathrm{T} 2$ trials were also removed if there was an error on T1 of these trials. Next, mean RTs for correct responses and percentage of errors were calculated for each of the conditions. Because there was no indication of a speed-accuracy tradeoff, our statistical analysis focuses on RTs.

Mean RTs for T1 and T2 as a function of SOA and T2 compatibility are shown in Figure 2 and Table 1. Table 1 also presents the error rates.

Task 1. The effect of SOA on RTs, though small, was significant $[F(3,72)=8.7, p<.01]$. This pattern of results is common to many PRP studies and does not seem to affect the $\mathrm{T} 2$ results.

Task2. A 4 (SOA) $\times 2$ (compatibility) repeated measures ANOVA revealed main effects of SOA $[F(3,72)=$ $221.0, p<.01]$ and compatibility $[F(1,24)=30.1 p<$

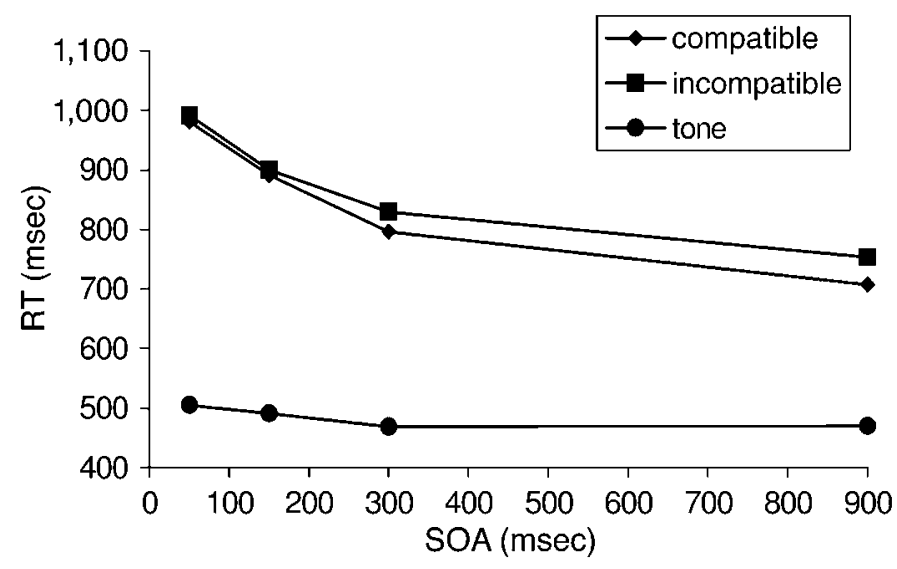

Figure 2. Mean reaction times (RTs, in milliseconds) for the first task (tone) and the second task (compatible vs. incompatible) in the first version of Experiment 1. 
Table 1

Mean Reaction Times (RTs, in Milliseconds) and Percentage of Errors (PEs)

for the Various Stimulus Onset Asynchrony (SOA, in Milliseconds)

Conditions in Task 1 (T1) and Task 2 (T2) of the Two Experiments

\begin{tabular}{|c|c|c|c|c|c|c|c|c|c|}
\hline \multirow[b]{3}{*}{ Experiment } & \multirow[b]{3}{*}{ Task } & \multicolumn{8}{|c|}{ SOA (msec) } \\
\hline & & \multicolumn{2}{|c|}{50} & \multicolumn{2}{|c|}{150} & \multicolumn{2}{|c|}{300} & \multicolumn{2}{|c|}{900} \\
\hline & & RT & $\mathrm{PE}$ & RT & $\mathrm{PE}$ & RT & $\mathrm{PE}$ & RT & $\mathrm{PE}$ \\
\hline \multirow[t]{4}{*}{1 , Version 1} & $\mathrm{~T} 1$ & 505 & 1.8 & 491 & 1.6 & 469 & 1.3 & 470 & 1.1 \\
\hline & $\mathrm{T} 2$, compatible & 980 & 0.8 & 891 & 0.7 & 796 & 1.1 & 707 & 0.2 \\
\hline & $\mathrm{T} 2$, incompatible & 991 & 1.4 & 900 & 1.5 & 830 & 1.4 & 753 & 1.3 \\
\hline & Spatial Simon effect & 11 & & 9 & & 34 & & 46 & \\
\hline \multirow[t]{4}{*}{1 , Version 2} & $\mathrm{~T} 1$ & 506 & 1.6 & 492 & 1.2 & 476 & 1.1 & 469 & 1.3 \\
\hline & $\mathrm{T} 2$, compatible & 990 & 1.6 & 903 & 1.1 & 826 & 0.9 & 720 & 0.8 \\
\hline & $\mathrm{T} 2$, incompatible & 995 & 1.6 & 916 & 1.6 & 841 & 1.8 & 776 & 1.8 \\
\hline & Spatial Simon effect & 5 & & 13 & & 15 & & 56 & \\
\hline \multirow{4}{*}{ 2, Version 1} & $\mathrm{~T} 1$ & 540 & 2.2 & 531 & 1.4 & 525 & 0.9 & 533 & 1.1 \\
\hline & T2, compatible & 1,064 & 0.9 & 981 & 1.1 & 871 & 0.7 & 712 & 0.9 \\
\hline & $\mathrm{T} 2$, incompatible & 1,088 & 3.1 & 996 & 2.5 & 897 & 2.7 & 739 & 2.3 \\
\hline & Color Simon effect & 24 & & 15 & & 26 & & 27 & \\
\hline \multirow{4}{*}{ 2, Version 2} & $\mathrm{~T} 1$ & 455 & 1.1 & 444 & 1.8 & 444 & 1.5 & 450 & 1.5 \\
\hline & $\mathrm{T} 2$, compatible & 922 & 0.9 & 827 & 1.1 & 753 & 0.6 & 641 & 0.6 \\
\hline & $\mathrm{T} 2$, incompatible & 946 & 2.9 & 852 & 2.1 & 776 & 1.7 & 664 & 1.5 \\
\hline & Color Simon effect & 24 & & 25 & & 23 & & 23 & \\
\hline
\end{tabular}

.01]. RTs decreased significantly as the SOA between the two tasks increased and were shorter for compatible than for incompatible trials, indicating the occurrence of a reliable spatial Simon effect. Most important, the spatial Simon effect interacted significantly with SOA $[F(3,72)=8.3, p<.01]$, indicating underadditivity of the spatial Simon effect with the SOA.

The color manipulation in Experiment 2 required the use of colored rather than gray squares. To ensure that underadditivity of the spatial Simon effect can be obtained with colored squares, we first ran a second version of Experiment 1 in which we tested 25 additional participants on the same task as in Version 1, except that in Version 2 the left and right squares were red and green, respectively. The results, shown in Figure 3 and Table 1, were virtually identical to those of Version 1 : The effect of SOA on T1 was significant $[F(3,72)=5.6, p<.01]$. A $4(\mathrm{SOA}) \times 2$ (compatibility) repeated measures ANOVA on T2 revealed significant effects of SOA $[F(3,72)=118.6, p<.01]$ and compatibility $[F(1,24)=$ $12.1 p<.01]$. The SOA $\times$ compatibility interaction was also significant $[F(3,72)=9.6, p<.01]$.

Experiment 1 fully replicated previous findings (Lien \& Proctor, 2000; McCann \& Johnston, 1992). In Experiment 2, a similar design was used to examine the interaction of a color Simon effect with the PRP effect.

\section{EXPERIMENT 2}

In Experiment2, we embedded a color Simon effect in the PRP paradigm. The participants responded to the identity of a central target shape by touching one of two

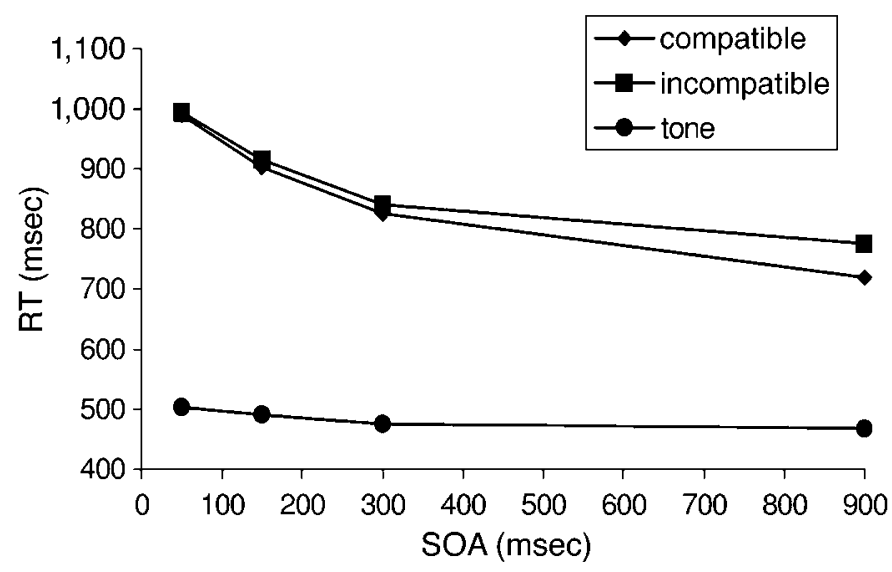

Figure 3. Mean reaction times (RTs, in milliseconds) for the first task (tone) and the second task (compatible vs. incompatible) in the second version of Experiment 1. 


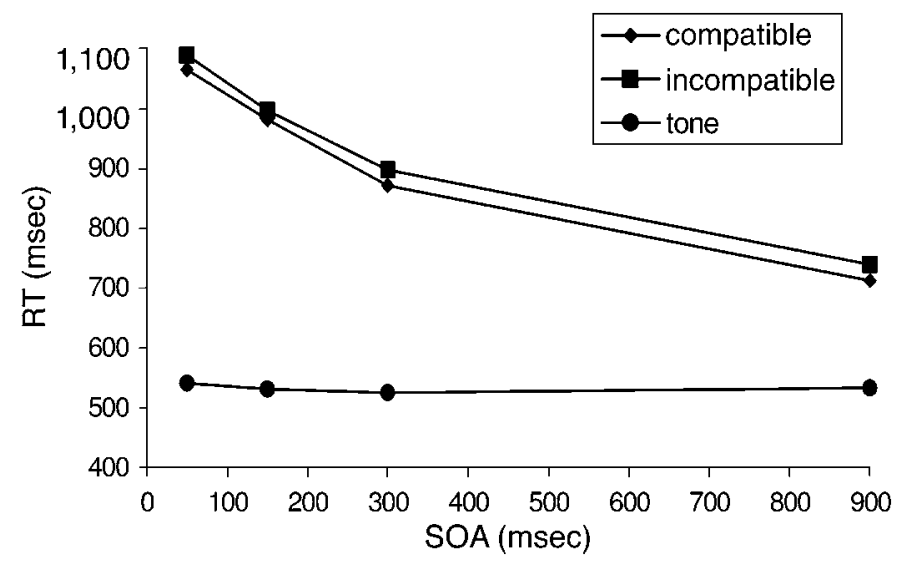

Figure 4. Mean reaction times (RTs, in milliseconds) for the first task (tone) and the second task (compatible vs. incompatible) in the first version of Experiment 2.

colored squares presented on a touch screen. The target's color was irrelevant to the task. Because this is the first demonstration of a color Simon task, we used two versions with slightly different T2s. The first version included the same letter task as in Experiment 1. The second version included a digit task. The target's color and the color of its assigned response square either matched (the compatible condition) or did not match (the incompatible condition). The color Simon effect was evaluated by comparison of the results of the compatible and incompatible conditions. Because the experiment was very similar to Experiment 1, only the aspects in which it differed from Experiment 1 are described.

\section{Method}

Participants. Fifty students, divided into two equal groups, participated in two separate sessions. One group performed the letter task, and the other performed the digit task.

Apparatus and Stimuli. The T2 central targets consisted of the letters $\mathrm{X}$ and $\mathrm{O}$ (Version 1 ) or the digits 3 and 4 (Version 2), colored in red or green. The digits subtended approximately $1.43^{\circ}$ of visual angle in length and $0.72^{\circ}$ in width. The left response square was green, and the right response square was red.

Design. T2 was either a letter task (as in Experiment 1) or a digit task. The color of the target shape was either red or green and changed randomly from trial to trial. The participants responded to $X$ (Version 1 ) or 3 (Version 2) by touching the left green square, and they responded to $\mathrm{O}$ (or 4) by touching the right red square. This design created two conditions. In the compatible condition, the target color matched the color of its assigned response (e.g., the digit 3, assigned to the green button, was presented in green). In the incompatible condition, the target color did not match the color of its assigned response (e.g., the 3 was presented in red). As was the case for Experiment 1 , this $4(\mathrm{SOA}) \times 2$ (auditory target) $\times 2$ (second task target) $\times 2$ (compatibility) factorial design resulted in 32 different displays.

\section{Results and Discussion}

We used the same type of analysis as in Experiment 1. Error rates and mean RTs for the various conditions are shown in Table 1. Mean RTs for T1 as a function of SOA

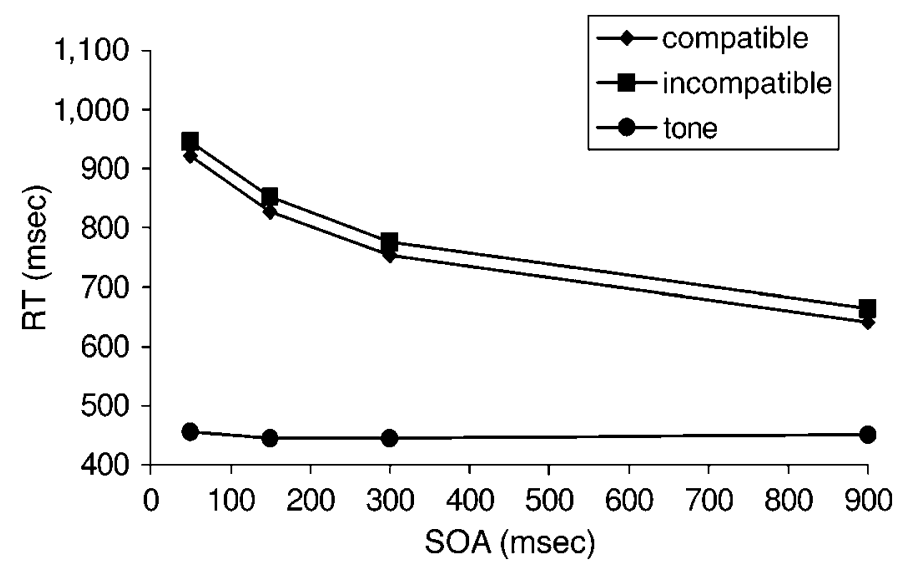

Figure 5. Mean reaction times (RTs, in milliseconds) for the first task (tone) and the second task (compatible vs. incompatible) in the second version of Experiment 2. 
and those for $\mathrm{T} 2$ as a function of both SOA and compatibility conditions are graphed in Figures 4 (Version 1) and 5 (Version 2).

Task 1. The effect of SOA on RTs was not significant in either Version 1 or Version $2[F(3,72)=1.1, p>.05$, and $F(3,72)=1.3, p>.05$, respectively].

Task 2. A 4 (SOA) $\times 2$ (compatibility) repeated measures ANOVA revealed a significant effect of SOA on $\mathrm{T} 2$ for both versions $[F(3,72)=186.9, p<.01$, and $F(3,72)=157.8, p<.01$, respectively $]$, showing the standard PRP effect. The color Simon effect was also significant for both Version 1 and Version $2[F(1,24)=$ $22.0, p<.01$ and $F(1,24)=27.6, p<.01$, respectively]. RTs were shorter for compatible than for incompatible trials. Most important, the color Simon effect did not interact significantly with SOA in either version $[F(3,72)<$ 1 for both versions]. The color Simon effect was additive with the SOA. This pattern of results is very different from that of the spatial Simon effect, which is underadditive with SOA.

To further compare the results of Experiments 1 and 2, we conducted a 2 (experiment) $\times 2$ (compatibility) $\times 4$ (SOA) mixed design ANOVA. For this analysis, we treated the two versions of Experiment 1 as a single experiment. Likewise, we treated the two versions of Experiment 2 as a single experiment. The main effects of SOA and compatibility were significant $[F(3,294)=621.3$ and $F(1,98)=84.6$, respectively, both $p \mathrm{~s}<.01]$, as was the SOA $\times$ compatibility interaction $[F(3,294)=9.9, p<$ $.01]$. Most important, the experiment $\times$ compatibility $\times$ SOA interaction was significant $[F(3,294)=7.6, p<$ $.01]$. This analysis clearly suggests that the relation between compatibility and SOA was different in the two experiments, being underadditive in Experiment 1 and additive in Experiment 2. Note also that the experiment effect did not approach significance $(F<1)$, ruling out any explanation based on differential difficulty of the two experiments.

\section{GENERAL DISCUSSION}

The underadditivity of the spatial Simon effect is a consistent notable exception in the PRP literature for T2s involving response selection manipulation. Our findings demonstrate that this exception is not due to Simon effects in general but rather may be unique to the spatial version. These results suggest that space plays a unique role in response selection processes.

Two hypotheses have previously been suggested to explain the underadditivity of the Simon effect. We first describe these two hypotheses and how they can possibly account for our data. We then propose a novel hypothesis that provides a different perspective on the role of space in response selection.

According to the decay hypothesis (McCann \& Johnston, 1992), irrelevant features of the target are activated with the presentation of the stimulus but decay rapidly. By the time participants respond to $\mathrm{T} 2$ in the small SOAs (in which RTs are longer because of the PRP effect), the target's spatial code has already decayed and no longer interferes with the response. Two lines of evidence support the decay hypothesis. First, creating a delay between the presentation of the target and response selection greatly reduces the Simon effect (Hommel, 1993). Presumably, by the time response selection occurs in these situations, the spatial code of the stimulus had already largely dissipated. Second, distribution analysis of RTs for the spatial Simon effect reveals that the effect is largest at the shortest RTs and decreases as RT increases. This analysis indicates that as the temporal distance be-

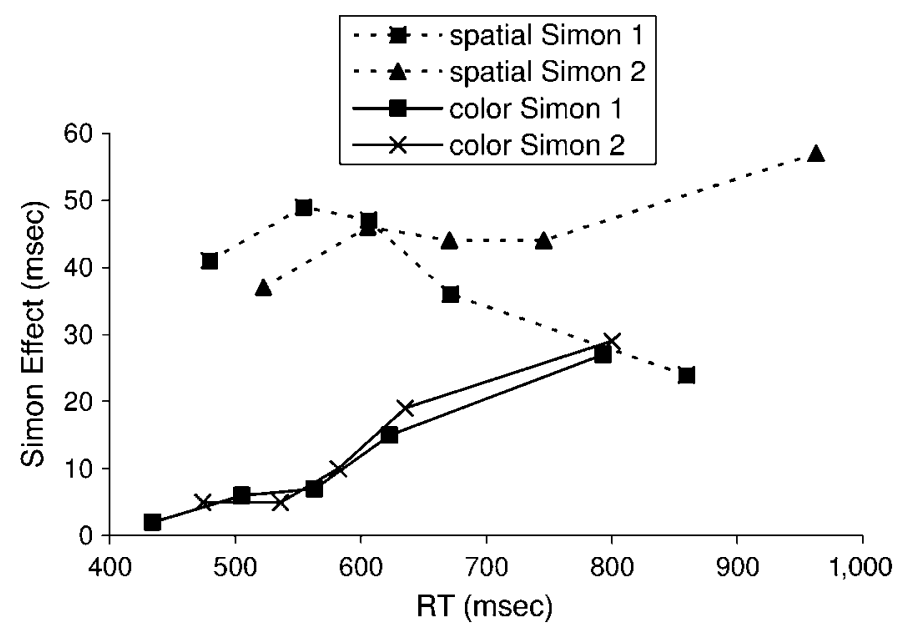

Figure 6. Reaction time (RT, in milliseconds) distribution analyses of a single task for the two versions of the spatial Simon task and for the two versions of the color Simon task. 
tween the stimulus onset and the response selection increases, the spatial Simon effect decreases (De Jong, Liang, \& Lauber, 1994; Hommel, 1997).

The decay hypothesis may incorporate our findings by assuming that decay is specific to spatial representations. This explanation predicts that if we apply the RT distribution analysis we should always find a decreasing effect, with longer RTs for the spatial Simon effect. Conversely, we should not find this pattern in the color Simon effect. In our study, we conducted two versions of the spatial Simon effect (Experiment 1) and two versions of the color Simon effect (Experiment 2). In each version, we tested 10 participants on T2 by itself. We did an RT distribution analysis for these tasks (Figure 6), dividing the RT distributions for the two compatibility conditions of each version into five quintiles and examining the Simon effect for each quintile. The color Simon effect did not decrease with longer RTs, as was predicted by the decay hypothesis. However, the spatial Simon effect, in which the participants responded by touching colored squares, did not show this pattern either. Lien and Proctor (2000) also found underadditivity of the spatial Simon effect without observing a decay of the effect. We do not have an explanation for the inconsistent results of the spatial Simon effect as a function of RT quintiles. This issue requires further research. Our main point is that the relation between the Simon effect and SOA is consistent, irrespective of the RT distribution analysis. That is, underadditivity is observed in the spatial Simon effect but not in the color Simon effect. Although our findings do not rule out some influence of decay on the spatial Simon effect, they suggest that other factors contribute significantly to underadditivity of the spatial Simon effect in the PRP paradigm.

The response activation hypothesis (Lien \& Proctor, 2000, following Hommel, 1998) distinguishes between activation and selection within response processes. The former occurs automatically, and its launching for T2 is not affected by T1 in the PRP paradigm. The latter cannot be performed in parallel for two tasks and is therefore postponed for T2 in the small-SOA conditions of the PRP paradigm. Lien and Proctor hypothesized that the Simon effect is due to response activation rather than to response selection processes. Because response activation processes occur for $\mathrm{T} 2$ irrespective of $\mathrm{T} 1$, they can also be resolved irrespective of $\mathrm{T} 1$, leading to underadditivity of the effect. To account for our findings, the response activation hypothesis would have to assume that automatic response activation is unique to spatial representations. Note, however, that Hommel (1998) observed automatic activation of response selection for nonspatial tasks as well.

We propose a third hypothesis, inspired by the unique role of space both in response selection processes, documented here, and in visual attention. We rely on two sets of previous findings. First, Pashler (1991) showed that visual attention can operate for $\mathrm{T} 2$ concurrently with T1 performance. Second, selection by visual attention can be accomplished by focusing on the location of motoric responses as well as on their input (see, e.g., Craighero, Fadiga, Rizzolatti, \& Umiltà, 1999; Hommel \& Schneider, 2002). We hypothesize that the underadditivity of the spatial Simon effect also results from the operation of visual attention. The Simon conflict occurs when participants map the input target (e.g., shape) to its response, which activates its location, while the irrelevant location of the target stimulus simultaneously activates the location of the alternative response. We hypothesize that in the spatial Simon situation, visual attention (which can operate in the small-SOA conditions for T2; cf. Pashler, 1991) can either focus on the location of the required response (cf. Craighero etal., 1999) or inhibit the alternative location activated by the irrelevant location of the target, thereby resolving the conflict. This can be done because visual attention operates on spatial representations. However, it can be used only to resolve spatial conflicts, such as those that arise in the spatial Simon effect. Attention does not have a direct connection to nonspatial representations (e.g., color representations) and cannot be used during the small-SOA conditions to resolve nonspatial conflicts such as that which arises in the color Simon task.

Further research is required to distinguish between these hypotheses. Any hypothesis, however, must include the unique role of space in response selection processes, as has been documented for the first time in our study.

\section{REFERENCES}

BAYlis, G. C., \& Driver, J. (1992). Visual parsing and response competition: The effect of grouping factors. Perception \& Psychophysics, 51, 145-162.

Cohen, A., \& Magen, H. (2004). Hierarchies of attention and action. In G. Humphreys \& J. Riddoch (Eds.), Attention in action (pp. 27-67). Hove, U.K.: Psychology Press.

Cohen, A., \& Shoup, R. (1997). Perceptual dimensional constraints on response selection processes. Cognitive Psychology, 32, 128-181.

Craft, J. L., \& Simon, J. R. (1970). Processing symbolic information from a visual display: Interference from an irrelevant directional cue. Journal of Experimental Psychology, 83, 415-420.

Craighero, L., Fadiga, L., Rizzolatti, G., \& Umiltà, C. (1999). Action for perception: A motor-visual attentional effect. Journal of Experimental Psychology: Human Perception \& Performance, 25, 1673-1692.

De Jong, R., Liang, C.-C., \& Lauber, E. (1994). Conditional and unconditional automaticity: A dual-process model of the effects of spatial stimulus-response correspondence. Journal of Experimental Psychology: Human Perception \& Performance, 20, 731-750.

Hommel, B. (1993). The relationship between stimulus processing and response selection in the Simon task: Evidence for a temporal overlap. Psychological Research, 55, 280-290.

Hommel, B. (1997). Interactions between stimulus-stimulus congruence and stimulus-response compatibility. Psychological Research, 59, 248-260.

Hommel, B. (1998). Automatic stimulus-response translation in dualtask performance. Journal of Experimental Psychology: Human Perception \& Performance, 24, 1368-1384.

Hommel, B., \& Schneider, W. X. (2002). Visual attention and manual response selection: Distinct mechanisms operating on the same codes. Visual Cognition, 9, 392-420.

Kornblum, S., HasbroucQ, T., \& Osman, A. (1990). Dimensional overlap: Cognitive basis for stimulus-response compatibility-a model and taxonomy. Psychological Review, 97, 253-270. 
Lamy, D., \& TsaL, Y. (2001). On the status of location in visual attention. European Journal of Cognitive Psychology, 13, 305-342.

Lien, M.-C., \& Proctor, R. W. (2000). Multiple spatial correspondence effects on dual-task performance. Journal of Experimental Psychology: Human Perception \& Performance, 26, 1260-1280.

Lu, C.-H., \& Proctor, R. W. (1995). The influence of irrelevant location information on performance: A review of the Simon and spatial Stroop effects. Psychonomic Bulletin \& Review, 2, 174-207.

Maruff, P., Danckert, J., CAMPlin, G., \& Currie, J. (1999). Behavioral goals constrain the selection of visual information. Psychological Science, 10, 522-525.

McCAnN, R. S., \& Johnston, J. C. (1992). Locus of the single-channel bottleneck in dual task interference. Journal of Experimental Psychology: Human Perception \& Performance, 18, 471-484.

Meyer, D. E., \& Kieras, D. E. (1997). A computational theory of executive cognitive processes and multiple-task performance: Part 1. Basic mechanisms. Psychological Review, 104, 3-65.

Pashler, H. (1991). Shifting visual attention and selecting motor responses: Distinct attentional mechanisms. Journal of Experimental Psychology: Human Perception \& Performance, 17, 1023-1040.

Pashler, H. (1994). Dual task interference in simple tasks: Data and theory. Psychological Bulletin, 116, 220-244.

PAshler, H., \& Johnston, J. C. (1989). Chronometric evidence for central postponement in temporally overlapping tasks. Quarterly Journal of Experimental Psychology, 41A, 19-45.
Posner, M. I., Snyder, C. R. R., \& Davidson, B. J. (1980). Attention and the detection of signals. Journal of Experimental Psychology: General, 109, 160-174.

SCHWEICKERT, R. (1980). Critical-path scheduling of mental processes in a dual task. Science, 209, 704-706.

Tsal, Y., \& Lavie, N. (1993). Location dominance in attending to color and shape. Journal of Experimental Psychology: Human Perception \& Performance, 19, 131-139.

WELFORD, A. T. (1952). The "psychological refractory period" and the timing of high-speed performance: A review and a theory. British Journal of Psychology, 43, 2-19.

\section{NOTE}

1. There is good evidence that color flankers that match or do not match an irrelevant color of a target shape do not affect behavior (see, e.g., Cohen \& Shoup, 1997; Maruff, Danckert, Camplin, \& Currie, 1999). Thus, the existence of a color effect in our design must be due to its association with the response, as is the case for a location effect in the spatial Simon effect.

(Manuscript received April 21, 2004; revision accepted for publication August 17, 2004.) 